\title{
Corona at Large Coated Electrodes
}

\author{
Mats Larsson ${ }^{1}$, Olof Hjortstam ${ }^{1}$, Håkan Faleke ${ }^{1}$, Liliana Arevalo ${ }^{2}$, Dong Wu ${ }^{2}$, Li Ming ${ }^{1}$ \\ ${ }^{I} A B B$ Corporate Research, Västerås, Sweden \\ ${ }^{2} A B B$ HVDC, Ludvika, Sweden
}

\begin{abstract}
In geometries relevant form HVDC applications where large electrodes and large air gaps are utilized, the observed corona can be quite different from geometries studied in the literature where needles or wires are used as high voltage electrodes. Corona discharges at large electrodes often initiates when the electric field on the electrode surface appears lower than the critical electric field strength, $2.4 \mathrm{kV} / \mathrm{mm}$. Surface contamination of the electrode has been pointed out as the reason for such discharge events. Our experimental results indicate that one possible way to prevent such corona is to coat the electrode with an insulating material, such as epoxy or oxide layers. It seems that the layer separates any corona inducing particle from the electrode, which in turn hinders the corona to form. However, as the layer breaks down and gets punctured, the corona preventing propertied disappears and corona forms easily. We conclude that as long as the layer doesn't get punctured, coating electrodes with insulating material is preventing corona to initiate at electrical fields below the critical electric field, as given by the electrode geometry. In contrast to positive polarity, for negative polarity the epoxy coating could withstand high electric fields without breaking down.
\end{abstract}

\section{Introduction}

DC corona for UHVDC systems has become a new challenge for the design of insulation due to the high Efield on the screen electrodes, which is a result from the UHV level and compact design requirements. A proper design of screen electrodes allows reduction of the air clearances while simultaneously minimize, or avoid, corona during service. A better understanding of the corona inception of large electrodes and to find out possible measures to increase the corona inception voltage levels are therefore of major concern.

Historically, corona discharges in air have been extensively studied. Since relatively low voltages then are required, the geometries studied have typically been laboratory setups utilizing sharp needles or wires as high-voltage electrodes [1, 2]. In such experiments, corona inception voltages are usually quite comparable for the AC and DC case. In contrast, relatively limited research on corona formation has been performed on larger electrodes and at higher voltage levels and at such conditions, the $\mathrm{AC}$ and $\mathrm{DC}$ case could differ quite significantly.

The laplacian electric field in lab setups can easily be calculated taking the electrode and the earth plane geometry into account. For large electrodes and large gaps, it can to the first approximation be used at as a tool for estimating when corona discharges starts. Corona occurs when the inhomogeneous electric field is sufficiently high in a volume large enough to start an electron avalanche [1, 2]. In order to develop any corona the field must accordingly be larger than the critical electric field of about $2.4 \mathrm{kV} / \mathrm{mm}$ for air, where there is net ionization [1]. So far, the discussion is based on perfect smooth electrode surfaces without any defects or protrusions that alter the electric field. For large electrodes, however, corona discharges can occur also when the geometrical electric field on the electrode surface is lower than the critical field strength, i.e. below $2.4 \mathrm{kV} / \mathrm{mm}$. A few published studies on DC corona inception on relatively large electrodes, such as big spheres and toroids together with long air-gaps show that corona inception can occur at voltages below the voltage that corresponds to the critical geometrical field $[3,4,5,6,7]$.

One explanation to the corona formation is that there are irregularities or contamination on the electrode surface that enhances the field in certain spots and therefore induce corona at those positions. Accordingly, in the previous experimental studies [6], steady glowing weak corona under negative DC voltage was identified by an UV camera. It is known that dust particles and dirt on the electrode surfaces can lead to corona at lower voltages than for the voltage where geometrical corona appears [8]. The authors of Ref [5] claims that dust particles from air or the floor play a major role for the observed corona inception voltage.

Based on the discussion above, we would like to identify two different classes of corona. The first type is geometrical corona, which refers to corona when the geometrical field becomes large enough to cause avalanche onset. Such corona is determined on the macroscopic shape of the electrode and a characteristic for geometrical corona is that the corona discharges is not located at the same point on the electrode at every discharge, instead the discharges appear at seemingly random spots around the highly stressed part of the electrode. The geometrical corona show quite strong discharges, often much more than $1000 \mathrm{pC}$, very loud and is easily detected by UV camera.

The second type is field enhanced corona, which refers to corona that is caused by some surface irregularity that enhance the field to such degree that corona is generated. Such corona can occur at lower voltages than the geometrical corona due to the field enhancement. Under negative polarity the typical field enhanced corona is a steady glowing spot which stationary on the 
electrode surface. The discharge can be very small (below our detection limit of $8 \mathrm{pC}$ ) but also significantly larger, up towards $1000 \mathrm{pC}$. The object initiating the field enhanced corona could be dust particles, surface roughness/properties and other protrusions caused by contaminations.

\section{Experimental details}

The corona discharges were studied experimentally with the electrodes suspended in the center of a cage, as shown in Figure 1. The cage floor and walls was made of perforated sheet metal, the walls were $2.5 \mathrm{~m}$ high and the diameter was $4 \mathrm{~m}$. A small opening was made in cage wall in order to access the test object optically with a UV sensitive camera. The typical DC voltage application scheme was to increase with $20 \mathrm{kV}$ steps every 2 minutes. When a sudden change in the corona pattern appeared, the increase might be stopped in between the two predetermined voltage levels. The tested electrodes in this study were toroids with outer diameter of $600 \mathrm{~mm}$ and a tube diameter of $100 \mathrm{~mm}$.

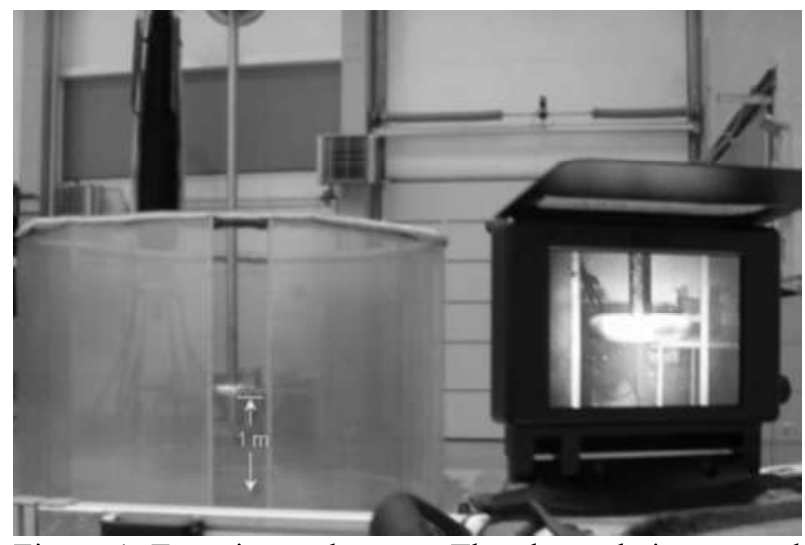

Figure 1: Experimental set up. The electrode is centered in the cage; the gap distance in this photo is $1 \mathrm{~m}$. The display of the UV camera is shown to the right.

\subsection{UV camera}

As depicted in Figure 1, a UV sensitive video camera was used to monitor the light emission associated with corona discharges. The camera was a DayCor classic corona camera manufactured by Ofil Ltd. It consists of a dual optic system providing a combined visual and UV sensitive image simultaneously, see Figure 2. Previous tests by $\mathrm{ABB}$ Corporate Research have shown that the sensitivity to corona is the highest available on the market. The UV detection was used to identify the local small glowing corona spots but also to monitor the locations of the geometrical corona discharges.

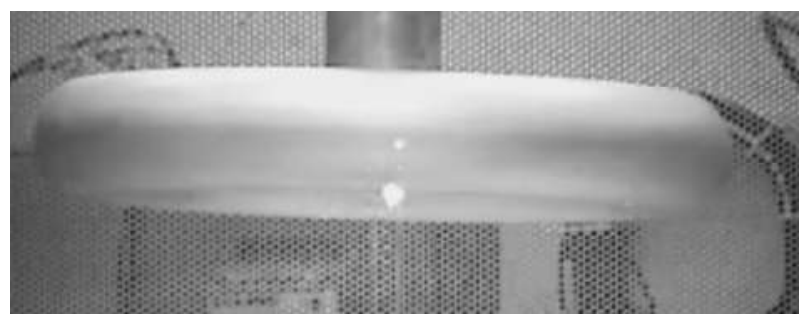

Figure 2: Image from the UV sensitive camera, illustrating a field enhanced corona spot on the lower front part of the electrode.

\subsection{Electrical detection}

The cage was insulated from the floor by a thin plastic sheet. PD was then measured between the cage and ground with a Haefely quadripole 568 as the shunt. The signal was filtered by a Krohn-Hite 3103 a tunable band pass filter, with low cut off at $150 \mathrm{~Hz}$ and high cut off at $190 \mathrm{kHz}$ before sampled by a Yokogawa DL850 scopecorder and transferred to the computer. The coupling capacitor of the quadripole was set to $10 \mathrm{nF}$ and the current to $2 \mathrm{~mA}$ by optimizing the signal to noise ratio with a calibration pulse applied on the test object. We were able to reach a noise level of $8 \mathrm{pC}$ with the described setup.

\subsection{Audible detection}

The noise from the corona was monitored by listening directly during testing and sometimes also using a microphone mounted in a parabola to amplify the noise signal. The audible detection is of course very dependent on the circumstances of the test and is therefore not objective. The method has however still proven useful to study corona discharges. Audible and electrical detection was the major methods used to register geometrical corona, before starting to record the corona with the UV camera.

\section{Results}

As a first step the voltage level of the geometrical corona was determined. The geometrical corona doesn't have a preferable spot, but are instead random in the region of highest electrical stress. By identifying such activities with the UV camera and by simultaneously confirming that as PD levels are above $1000 \mathrm{pC}$, the inception level of geometrical corona were identified.

Several identical electrodes were tested, a few with normal polished aluminum surface, a few with different coating and one with fine polished surface and one sanded, rougher, surface. Even though the surface properties were quite different, the inception of geometrical corona for all those electrodes was very similar without any special surface treatment that singled out as significant. The standard deviation was $2,5 \%$ of the mean value of the corona inception, see Figure 3. 


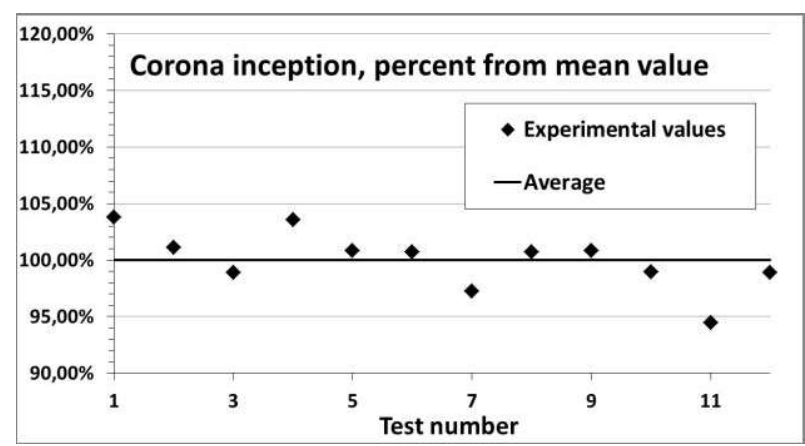

Figure 3: Geometrical corona inception for all electrodes tested.

While the geometrical corona is predictable, prediction of the field enhanced corona that occurs at lower electrical field is much more demanding. The inception of field enhanced corona could be as low as $50 \%$ of the geometrical corona. It was often only detected by the UV sensitive camera but as the voltage was ramped up; also electrical and audible detection was possible since the corona then increases in strength. The inception level is sorely dependent on the geometrical and electrical properties of the piece of dust/dirt or surface irregularity that in that test induce the corona, and therefore very hard to predict. After each measurement one could observe that the electrostatic DC field had attracted small particles and dust to the electrode, which in some cases, and in some cases not, had given rise to corona.

\subsection{Negative polarity epoxy coating}

One effective way to prevent the field enhanced corona at negative polarity that was established here, is to coat the electrode with an insulating material. One of the electrodes was coated with an epoxy layer that was about 250 microns thick and during a number of experiments with this epoxy coated electrode, the object was free from any glowing field enhanced corona. Dust could still be attracted to the electrode surface when the field is applied but they will not induce any glowing corona. Some very occasional small discharges were observed but no continuous as in the case of a bare electrode. One interpretation is that the any field enhancing contaminations on the electrode surface are insulated so no charges are supplied to feed the continuous discharge. The small discharges that occasionally were detected might also charge the insulating surface and thereby helped to screen the corona spot, preventing continued discharging. As shown in Figure 4, small metal wires were glued onto the epoxy surface in order to intentionally induce field enhanced corona. We could not observe any field enhanced corona from those spots under negative polarity, which is in sharp contrast to experiments with glued on wires on bare electrodes. The fact that we can't get field enhanced corona from the epoxy coated electrode even when putting a pointy object on its surface convincingly demonstrates the ability of the coating to prevent corona. However, when the field is high enough for the geometrical corona to start, the epoxy coating tends to get punctured, which results in a very active corona initiation point. The inception voltage for that point is much lower than the inception for geometrical corona.

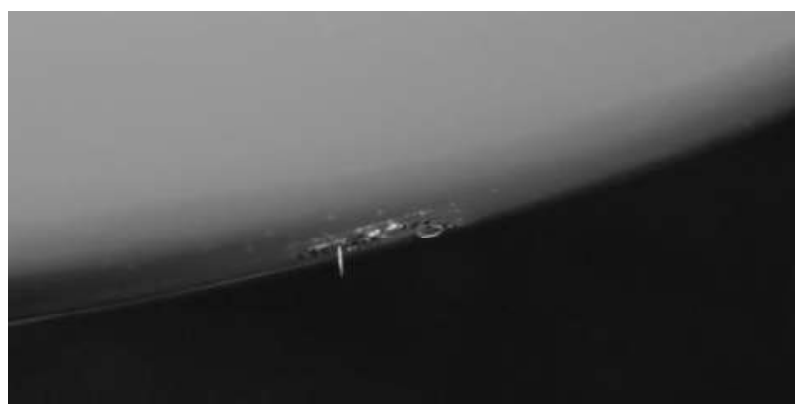

Figure 4: Epoxy coated electrode with a metallic wire glued on to its surface.

\subsection{Positive polarity epoxy coating}

For positive polarity, the breakdown of the epoxy layer seems to be an even bigger problem. During our tests at positive polarity, the epoxy coating tends to break down and get punctured already at $80 \%$ of the geometrical corona inception. At one such punctured spot, the corona extinction voltage of a punctured epoxy can be as low as $40 \%$ of the geometrical corona. Tests with glued on metal wires were also performed at positive polarity which also resulted in punctured epoxy. An interesting observation is however that the epoxy did not puncture directly under the metal wire, instead the breakdown of the epoxy layer happen in the vicinity of the glued on wires. The region around the metal wire seems to get charged up and then breaks down at a spot where the epoxy has some local weakness.

\subsection{Anodized electrode}

A bare electrode has just a few nanometer of a naturally formed oxide layer, while an anodized aluminum surface has an oxide layer of at least several hundreds of nanometer. An electrode was anodized and tested in order to compare with the bare and epoxy coated electrodes. As the voltage was ramped up no glowing corona, as often seen on bare electrodes, was observed. However, there were observations of occasional discharges below the inception of geometric corona. Accordingly, the oxide layer seems to reduce the corona activity but is not able to totally avoid it.

\section{Discussion}

The geometrical corona is an effect of the properties of air in combination with the geometry of the equipment and can thus be controlled by design of the HVDC apparatuses. The field enhanced corona which appears at relatively low voltages must be avoided by other means. It is therefore important to identify the origin of the corona and study the possible routes to prevent it. Corona resulting from the macroscopic geometrical field is essentially unaffected by the microscopic 
surface structure. Electrodes with polished and buffed surfaces shows similar inception values as electrodes with more rough surfaces. Also electrodes with anodized and epoxy coating display the same typical values. The standard deviation of geometrical corona inception voltage was $2,5 \%$ of the mean inception voltage. The electric field at the geometrical corona inception voltage is close to the $3 \mathrm{kV} / \mathrm{mm}$ on the electrode surface which, as expected, gives corona discharges.

On the other hand, the well documented corona at voltage levels where the geometrical field doesn't exceeds the $2,4 \mathrm{KV} / \mathrm{mm}$ requires some kind of field enhancement in order to appear. The UV camera observation of small and steady glowing corona spots suggest that some local field enhancement occurs. Such field enhancement could have origin in dust/dirt on the surface, sharp features on the surface form the manufacturing, or some other kind of surface contamination. Our observations of the epoxy coated electrode, where the field enhanced corona could be eliminated fits well with a picture that such corona are related to the electrode surface or something on the electrode surface. By drastically change the surface properties, we were able to prevent field enhanced corona. However, the standard epoxy layer that was used in these tests got easily punctured during positive polarity but also under high negative polarity stress. Once the coating are punctured, that point effectively cause corona discharges.

Since the mobility of the electrons and the ions in air are very different, there is a significant difference between negative and positive corona. For the negative polarity, the electron avalanche develops away from the electrode. While for positive polarity the electron avalanche accelerated towards the electrode. This might explain the difference for the ability of the coating to withstand positive and negative corona. For all measurements, positive discharges are actually generally much stronger than the negative ones, at least up to the voltage level where geometrical corona starts. Moreover, the coating will inevitable be charged during these kind of tests. From the experiments with the glued-on metal wires, it was shown that the coating broke down at a position close to the wire but not directly under it. That observation suggests that the puncturing of the epoxy is due to charging and the resulting high electric field across the coating. Also, the charging probably helps to screen any corona spot on the surface and thereby prevent continuous discharge. Assuming that the coating can get more easily charged with electrons compared to positive ions would explain the difference in positive and negative polarity. Whether the positive and negative corona difference or the charging of the coating can explain the difference in strength against puncturing of the epoxy needs to be addressed in future studies.

The anodization process results a relatively thick oxide layer which also probably could have self-healing properties after puncturing, scratching etc. Since the anodized electrode does not totally prevent corona discharges at voltage lower than the geometric corona level but, on the other hand, does not show any glowing corona, it's properties lies somewhere between the bare and the epoxy coated electrode in terms of preventing field enhanced corona. Assuming that particles induce any corona below the geometric corona inception, our observations can be explained by the increased insulation layer thickness for the bare, anodized and epoxy coated electrode, respectively.

\section{Conclusion}

In the system of large electrodes and large gaps, geometrical DC corona is to large extent unaffected by the microscopic surface roughness. The often seen corona that occurs at lower voltages can be reduced and sometimes eliminated by coating the electrode with a dielectric material. However, the epoxy material that was tested here got punctured as the field got to high. This problem is severe for positive polarity, where the coating broke down at least $20 \%$ below the geometrical corona start. An electrode with anodized surface also showed promising results without the problem of puncturing. After this first test round, further investigations are needed.

\section{References}

[1] L. B. Loeb, Electrical Coronas Their Basic Physical Properties, University of California Press, Berkely and Los Angeles 1965

[2] M. Goldman and R. S. Sigmond, Corona and insulation, IEEE Transactions on Electrical Insulation Vol. Ei-17 No.2, April 1982

[3] K. Feser: Dimensioning of electrodes in the UHV range-illustrated with the example of toriod electrodes of voltage dividers, Haefely Publication, 1975

[4] G. Degli Esposti: Considerations on the maximum allowable surface gradients on the electrodes of the high voltage apparatus, Proceedings of ISH 1, Munich, Germany 1972

[5] I. Ovsyanko, J. Speck, S. Großmann, Dimensioning of screening electrodes for extremely high rated direct voltage, Proceedings of ISH 16, Johannesburg, South Africa2009

[6] L. Ming, M. Larsson, A. Maxwell, H. Faleke, D. $\mathrm{Wu}$ and B. Jacobson, "Negative DC corona inception on large electrodes in a large air gap", Proceedings of ISH 17, Hannover, Germany, 2011

[7] I. Ovsyanko, J. Speck, S. Großmann, Dimensioning and testing of DC insulations in air, Proceedings of ISH 17, Hannover, Germany, 2011

[8] L. Ming, M. Larsson, A. Maxwell, H. Faleke and D. Wu, DC corona inception with contaminated high voltage electrode, Proceedings of Nordis 22, Tampere, Finland, 2011 